\title{
Hemocompatibility of stent materials: alterations in electrical parameters of erythrocyte membranes
}

This article was published in the following Dove Press journal:

Vascular Health and Risk Management

20 March 2012

Number of times this article has been viewed

\author{
A Basoli' \\ C Cametti ${ }^{2}$ \\ F Ginnari Satriani \\ P Mariani ${ }^{3}$ \\ P Severino ${ }^{3}$ \\ 'Department of Surgery, "P Stefanini," \\ University of Rome "La Sapienza," \\ Rome, Italy; 'Department of Physics, \\ University of Rome "La Sapienza," \\ Rome Italy; ${ }^{3}$ Department of Internal \\ Medicine, University of Rome \\ "La Sapienza," Rome, Italy
}

Background: It is presently unknown if stents used in the correction of artery stenosis are fully hemocompatible or if their implantation causes alterations at the level of the plasma membrane in red blood cells.

Methods: We addressed this important issue by measuring the passive electrical properties of the erythrocyte membrane before and after stent insertion by means of dielectric relaxation spectroscopy in the radiowave frequency range in a series of patients who were undergoing standard surgical treatment of arterial disease.

Results: Our findings provide evidence that full hemocompatibility of stents has not yet been reached, and that there are some measurable alterations in the passive electrical behavior of the red blood cell membrane induced by the presence of the stent.

Conclusion: It is possible that these changes do not have any physiological significance and simply reflect the intrinsic variability of biological samples. However, caution is urged, and the technique we describe here should be considered when investigating the hemocompatibility of a medical device at a cell membrane level.

Keywords: hemocompatibility, stent, arterial disease, cell membrane

\section{Introduction}

A biomaterial can only be considered truly biocompatible when it satisfies two conditions. First, the physicochemical properties of the biomaterial should remain unchanged over time, so that it can exert its function without alteration. Second, the biomaterial should not cause any change in the biological system with which it comes in contact, ie, it must do what it is supposed to do without provoking any unwanted modification in the host system. Therefore, the biomaterial must not only be completely inert, but must also not have any undesirable effects. These two requirements are often, at least partially, in conflict, meaning that a completely inert biomaterial may be ineffective at the target to which it is directed, in the same way as a particularly active biomaterial may induce unwanted reactions. The key is to find an appropriate compromise in the evaluation of cost-benefit, considering the advantages conferred by the biomaterial versus the possible, and in many cases unavoidable, alterations it may provoke in the host system.

Biomaterials have become very popular medical devices. There are several definitions of biomaterials. In 1982, the National Institutes of Health determined that the word "biomaterial" means "any substance or any combination of substances different from drugs of a natural or synthetic origin, which could be used for any period of time 
by itself or as a part of a system which handles, increases or replaces any kind of tissue, organ or function of the body". It is certainly not easy, even keeping in mind the great variability of materials used in different applications, to find a parameter which verifies the biocompatibility of a biomaterial in an objective and measurable way. ${ }^{1,2}$

For devices which come into contact with blood, such as those used in antithrombogenic implants, hemocompatibility is necessary, and lack of it could lead to many clinical complications, including chronic inflammation or cytotoxic effects.

A significant proportion of cardiovascular disease is caused by clogging of the arteries due to buildup of cholesterol. Stenting is one of the most common procedures used to treat stenosis, and many devices for arterial intervention have been tested during the last 20 years. Multidisciplinary efforts have been put into stent research and, as a result, new designs and different materials and coatings have been proposed to improve the performance of these prostheses. Among these, polymeric-coated stents, heparin-coated stents, drug-eluting stents, and others warrant mention. ${ }^{3}$ However, medical complications, including stent thrombosis, cholesterol accumulation, restenosis, inflammation, and hyperplasia are not infrequent.

It is unknown if, in addition to these unwanted macroscopic reactions, other alterations could be present at the level of the cell membrane. Therefore, it is fundamental to understand the dynamic processes that regulate interaction between the foreign material and the host system at the molecular level. Because the stent is placed in direct contact with a layer of erythrocytes and blood platelets, and there is no barrier between them and the material itself, the best strategy for investigating the cytotoxicity and biocompatibility of these devices is to examine parameters that describe the electrical properties of the cell membrane. Among these, two parameters, both of an electrical nature, have relevance in the characterization of both the structure and function of the plasma membrane. These are membrane permittivity $\left(\varepsilon_{s}\right)$ and membrane conductivity $\left(\sigma_{s}\right){ }^{4}$ The former is related to the distribution of charges and/or polar groups across the membrane lipid bilayer, and the latter is a measure of the overall ionic transport occurring across the cell membrane through ion channels and pores. These parameters involve some of the most important characteristics of the cell membrane, as far as their electrical behavior is concerned.

Consequently, knowledge about these parameters offers the opportunity to determine if an alteration in the cell membrane has occurred, induced by interaction with the biomaterial with which it comes in contact. In other words, the membrane permittivity $\left(\varepsilon_{s}\right)$ and the membrane conductivity $\left(\sigma_{s}\right)$ can be used as parameters to judge the biocompatibility of stents at a microscopic level. The absence of changes in $\varepsilon_{s}$ and $\sigma_{s}$ after prosthesis insertion is a necessary prerequisite so that the material does not provoke any alteration in red blood cells, at least as far as their electrical properties are concerned.

It should be borne in mind that the cell membrane separates media of different electrical properties (the extracellular solution and the cytosol) and, consequently, under the influence of an external electric field, charge polarization takes place at the interface between these two media. In particular, at frequencies of $1-100 \mathrm{mHz}$, a cell suspension undergoes a marked dielectric relaxation effect due to interfacial polarization, known in the colloidal community as $\beta$ dispersion. ${ }^{5}$ Because of this effect, using an appropriate dielectric cell model, it is possible to determine the two aforementioned electrical parameters, ie, membrane permittivity $\left(\varepsilon_{s}\right)$ and membrane conductivity $\left(\sigma_{s}\right)$ and then characterize the electrical behavior of the cell membrane and ascertain if it is a site of possible alteration.

The dielectric properties of biological cell suspensions have been extensively studied since the beginning of the 20th century, ${ }^{6}$ and since then a variety of different biological cells have been investigated. . $^{5-9}$

In the present work, we measured the electrical parameters of red blood cell membranes, ie, permittivity $\left(\varepsilon_{s}\right)$, electrical conductivity $\left(\sigma_{s}\right)$, and conductivity $\left(\sigma_{p}\right)$ of the cytosol in patients who had undergone correction of arterial stenosis by means of a metallic stent in order to ascertain the full biocompatibility of these devices.

In the past, we have observed that different kinds of stents could induce remarkable alteration in the passive electrical behavior of erythrocytes, but our observation has been limited to a period of 30 days after surgery at the most. ${ }^{10-12}$ Here we have extended those investigations, and followed the electrical parameters of the cell membrane for a much longer period of time of up to 8 months. Our results confirm once again that full hemocompatibility is not yet reached, at least on the basis of the definition given earlier, and that a word of caution is necessary.

\section{Materials and methods \\ Materials}

Data were collected from 14 patients who underwent standard surgical treatment for arterial disease using a stainless steel BioMatrix stent (Biosensors Interventional Technologies 
Pte Ltd, Singapore). These devices offer a combination of an antirestenotic drug (a biodegradable polylactic acid polymer) and a highly flexible material, and are widely used for arterial implantation. The stents were implanted according to the guidelines of the US Food and Drug Administration, the American Association for Vascular Surgery, ${ }^{13}$ and the Society for Vascular Surgery. ${ }^{14}$ All patients were numbered in consecutive order. Individuals who had had major surgery or a blood transfusion within the previous 6 months were excluded from the study.

Blood samples were obtained from the patients immediately before and after (within a period of time of 4 months and 8 months, respectively) insertion of the stent. The erythrocytes were centrifuged at $1000 \times \mathrm{g}$ for 5 minutes. The supernatant and buffy coat were removed by aspiration. The cells were resuspended and washed in $10 \mathrm{~mL}$ of phosphatebuffered saline (145 $\mathrm{mM} \mathrm{NaCl}$ and $5 \mathrm{mM}$ phosphate, $\mathrm{pH}$ 7.32) three times. The hematocrit of the final erythrocyte suspension was measured and adjusted to a $\Phi$ of 0.20 . This choice is a reasonable compromise between the need to have a high value of the volume fraction $(\Phi)$, in order to increase the dielectric effect, and to have a volume fraction not too high in order to reduce cell-cell interactions, as necessary for the dielectric model we used. Dielectric measurements were completed within 1-2 hours of the start of preparation of each sample.

The present study was carried out using erythrocytes isolated from whole blood and then dispersed in physiological saline. It could be relevant to extend these dielectric measurements to platelet-rich plasma, rather than erythrocyte suspensions, given that blood platelets are central to the process of thrombosis.

\section{Dielectric measurements}

Measurements of the dielectric properties of the erythrocyte suspensions were carried out using commercially available Hewlett-Packard impedance analyzers, ie, model 4192A, in the frequency range from $1 \mathrm{kHz}$ to $10 \mathrm{MHz}$, and model $4291 \mathrm{~A}$, in the frequency range from $1 \mathrm{MHz}$ to $2 \mathrm{GHz}$, together with a sample measuring cell consisting of a short section of a $3 \mathrm{~mm}$ electrode space coaxial cable connected by an APC7 precision connector to the meter. The sample cell was kept at a temperature of $25.0^{\circ} \mathrm{C} \pm 0.1^{\circ} \mathrm{C}$ in a temperature-controlled bath. Each run in the frequency window investigated was taken three times and the datum points are the average of these runs. The overall uncertainty of the measured permittivity $\varepsilon_{s}(\omega)$ and the measured conductivity $\sigma_{s}(\omega)$ was $0.5 \%$ and $1 \%$, respectively. In order to avoid further thermal shock, dielectric measurements were carried out using the same temperature $\left(25^{\circ} \mathrm{C}\right)$ at which the samples were maintained during preparation.

\section{Dielectric model}

The dielectric relaxation data and, in particular, the dielectric dispersion occurring in the intermediate frequency range of the frequency window investigated, were analyzed within the usual heterogeneous system theory framework. ${ }^{4,5,15,16}$ From a dielectric point of view, a biological cell suspension can be considered as a collection of ellipsoidal particles uniformly dispersed in a continuous aqueous phase. ${ }^{5}$ In the case of a red blood cell, each particle is represented by an oblate ellipsoid of semi axes, $a_{0}$ and $b_{0}$, of complex dielectric constant $\varepsilon_{p}^{*}(\omega)=\varepsilon_{p}+\sigma_{p} / i \omega \varepsilon_{0}$, covered by a confocal shell of complex dielectric constant $\varepsilon_{s}^{*}(\omega)=\varepsilon_{s}+\sigma_{s} / i \omega \varepsilon_{0}$ and thickness $\mathrm{d}$, and immersed in an aqueous phase of complex dielectric constant $\varepsilon_{m}^{*}(\omega)=\varepsilon_{m}+\sigma_{m} / i \omega \varepsilon_{0}$. Here, $i=\sqrt{-1}, \omega$ is the angular frequency of the applied electric field, and $\varepsilon_{0}=8.9610^{-12} \mathrm{~F} / \mathrm{m}$ is the dielectric constant of free space.

Within this scheme, the cell membrane and the cytosol are characterized by three electrical parameters, ie, $\varepsilon_{s}$ (membrane permittivity), $\sigma_{s}$ (membrane conductivity), and $\varepsilon_{p}$ (intracellular permittivity). Dielectric spectra, recorded over a wide enough frequency interval, allow these electrical parameters to be evaluated appropriately, offering the opportunity to evaluate electrical behavior and any changes thereof in both the cell membrane and cytosol. ${ }^{17-19}$

Figure 1 shows a typical dielectric spectra for erythrocytes dispersed in phosphate-buffered saline at $25^{\circ} \mathrm{C}$. Permittivity (upper panel) and electrical conductivity (bottom panel) are shown separately, in the frequency range of $1 \mathrm{kHz}$ to $2 \mathrm{gHz}$. The whole dielectric spectra, within the framework of effective medium theory approximation, can be described by a relaxation function as follows:

$$
\mathcal{E}^{*}(\omega)=A \omega^{-\beta}+F\left(\varphi_{e f f}^{*}, \varphi_{m}^{*}, \varphi_{p e q}^{*}, \Phi\right)+f_{w}
$$

where $\varphi_{\text {eff }}^{*}$ and $\varphi_{m}^{*}$ represent the complex dielectric constant of the cell suspension and suspending medium, respectively, and $\varphi_{p e q}^{*}$ is the complex dielectric constant of the biological cell. The fractional volume of the cells in the suspension is denoted by $\Phi$. The three terms of equation 1 correspond to the three different relaxation processes observed in biological cell suspensions in this frequency range, ie, the electrode polarization effect (described by the function $A \omega^{-\beta}$ ), the interfacial polarization of heterogeneous systems (described 

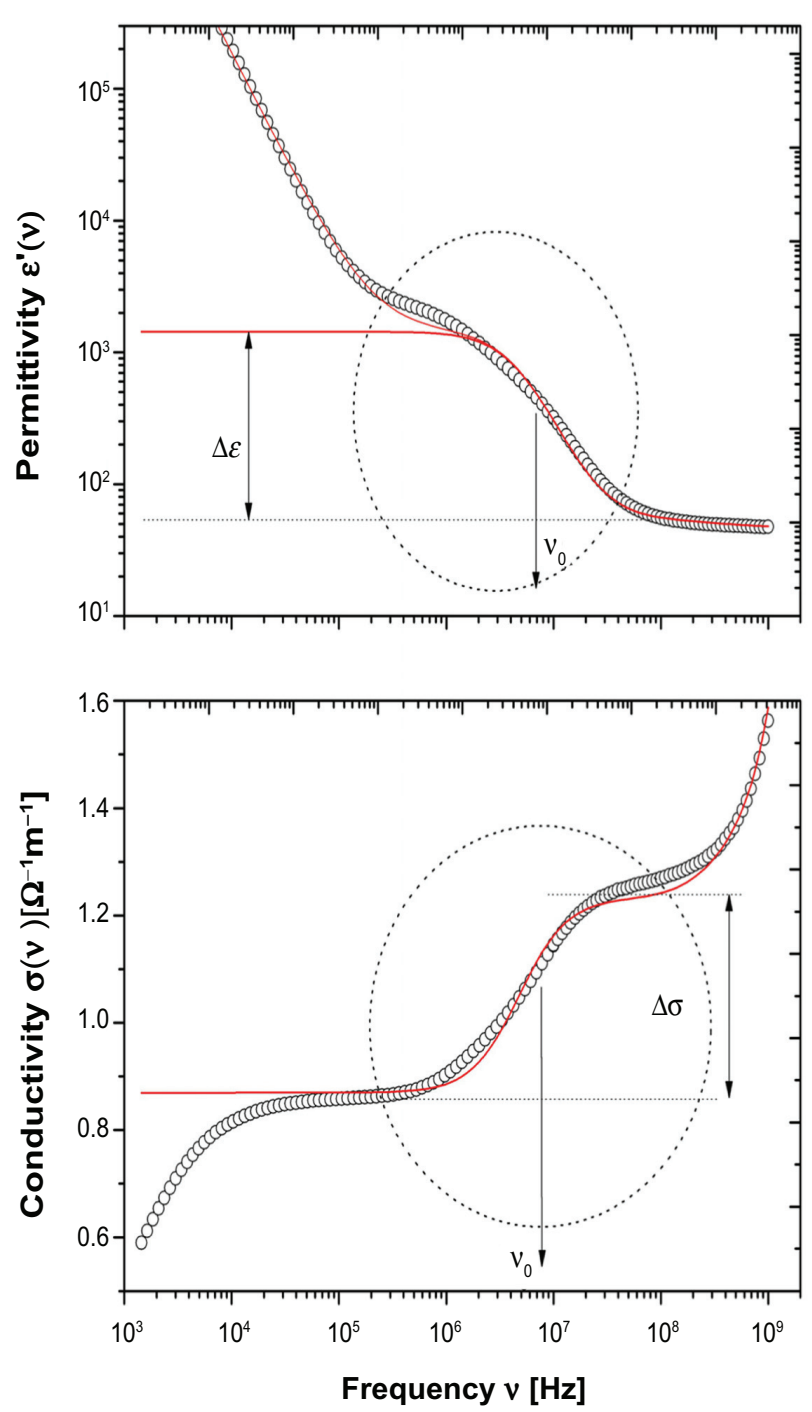

Figure I Typical dielectric spectra of erythrocytes in phosphate-buffered saline (hematocrit $\Phi 0.20$ ) at a temperature of $25^{\circ} \mathrm{C}$.

Notes: Upper panel shows permittivity $\varepsilon(\omega)$ and bottom panel shows electrical conductivity $\sigma(\omega)$. The dotted circle marks the relaxation region associated with the interfacial polarization due to the ion distribution at the cell membrane interface. From this relaxation region, the parameters $\varepsilon_{s}$ and $\sigma$, associated with the electrical characteristics of the cell membrane can be properly extracted. Full lines represent values calculated on the basis of the dielectric model used.

by the relaxation function, $F$ ), and the orientational relaxation in the aqueous phase (described by the relaxation function, $f_{w}$ ). The functional dependence of $F$ on the electrical parameters of the cell membrane, as demonstrated in the ellipsoidal single-shell model, is well known and has been reported in detail elsewhere. ${ }^{20,21}$

In this study, we focus our interest on dielectric dispersion (marked by a dotted circle in Figure 1) due to the interfacial polarization occurring because of accumulation of electrical charge at the interface between the cell membrane and the extracellular medium. The other dielectric dispersions, ie, the dielectric dispersion in the low-frequency tail and the one in the high-frequency tail of the frequency window investigated are due to different polarization mechanisms that do not involve, at least at first approximation, the presence of the cell membrane. These two other dielectric dispersions are beyond the scope of the present paper and are not discussed further.

\section{Statistical analysis}

The results are presented as the mean \pm standard deviation unless otherwise stated. Data within each group (the initial or later 4-month period) were analyzed by one-way analysis of variance followed by the Student-Newman-Keuls (SNK) test that makes pairwise comparison of groups after oneway analysis of variance has rejected the null hypothesis. The analysis has been performed at a significance level of $\alpha=0.01$, whereby the hypothesis of equality of the means is rejected when one-way analysis of variance produces a $P$ value less than $\alpha$.

\section{Results and discussion}

The dielectric dispersion considered here contains the pertinent information by which it is possible to extract the electrical parameters relevant to the electrical characterization of the cell membrane. They are membrane permittivity $\left(\varepsilon_{s}\right)$ and membrane conductivity $\left(\sigma_{s}\right)$, which represent the sign for possible alterations induced by the presence of the stent. Moreover, conductivity $\left(\sigma_{p}\right)$ of the cytosol can be obtained as well. We have omitted many of the details of the technical procedures used and the fitting algorithm that allows us to do this. Details of this are reported extensively elsewhere. ${ }^{5,9}$ Briefly, we employed the Levenberg-Marquardt algorithm ${ }^{15}$ for complex functions which allows the simultaneous fit of real and imaginary parts of the complex dielectric constant with the same set of free parameters. Adopting this standard methodology, 5,9 once the data were corrected for the electrode polarization effect, we derived the characteristic electrical parameters associated with the erythrocyte membrane, ie, membrane permittivity $\left(\varepsilon_{s}\right)$, membrane conductivity $\left(\sigma_{\mathrm{s}}\right)$, and conductivity of the intracellular medium $\left(\sigma_{p}\right.$, in the cytosol) for each of the samples investigated. These values were determined for each patient immediately before and after (within a period of time of 4 months and 8 months, respectively) insertion of the stent. These data are shown in Figures 2-4, reported as the difference between the values after and before treatment and normalized to the value before treatment.

As can be seen, all three parameters, referring to the entire sample investigated, showed marked alterations, in the sense 


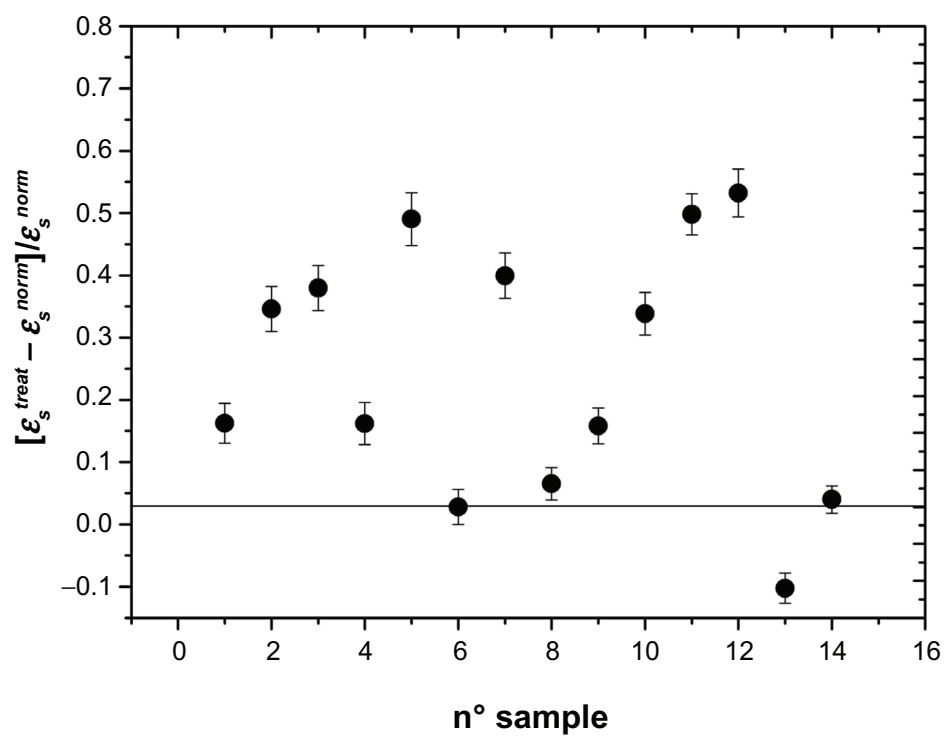

Figure 2 Change in membrane permittivity $\varepsilon_{s}$ normalized to the untreated value: $\left[\varepsilon_{s}^{\text {teat }}-\varepsilon_{s}^{\text {nom }}\right] / \varepsilon_{s}^{\text {nom }}$ of the erythrocyte membrane in different patients who underwent carotid artery stenting.

Note: Absence of any alteration is indicated by the horizontal line.

that we recorded a marked increase in these parameters after stent implantation. In the absence of any interaction, we could record values close to zero, marked by a horizontal line in Figures 2-4. In these figures, we found a remarkable scattering of the data. Given that we are reporting the normalized percentage change for each patient, the intrinsic variability of these parameters ascertainable in normal conditions in healthy people should be at least in part accounted for. In spite of this, our data show that, compared with initial measurements, insertion of the stent provokes a substantial increase in their values in all patients investigated, with the exception of one. The results were investigated by one-way analysis of variance to test for differences before and after stent insertion. At $P<0.01$, one-way analysis of variance showed that significant differences occurred between the groups. The second aspect we wanted to address was whether or not these alterations persist in time or if some type of recovery could take place. This issue has been considered in a previous paper, ${ }^{10,12}$ in which we reported measurements taken 8 days and one month after stent insertion. In that case, we

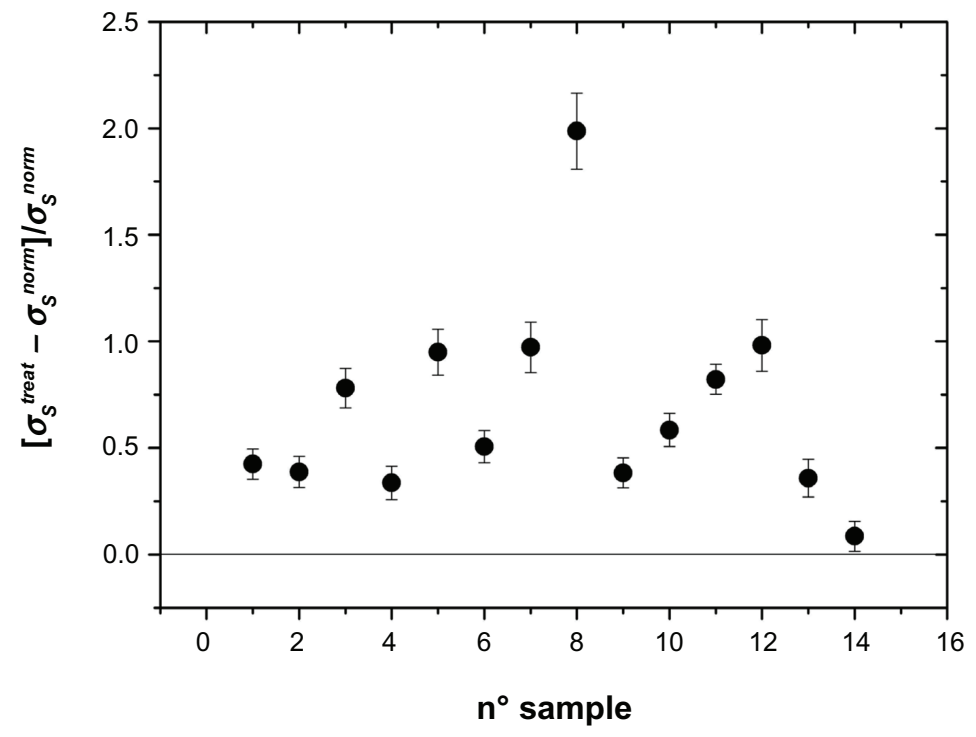

Figure 3 Change in membrane conductivity $\sigma_{s}$ normalized to the untreated value: $\left[\sigma_{s}^{\text {treat }}-\sigma_{s}^{\text {norm }}\right] / \sigma_{s}^{\text {nom }}$ of the erythrocyte membrane in patients treated for carotid artery stenting.

Note: Absence of any alteration is indicated by the horizontal line. 


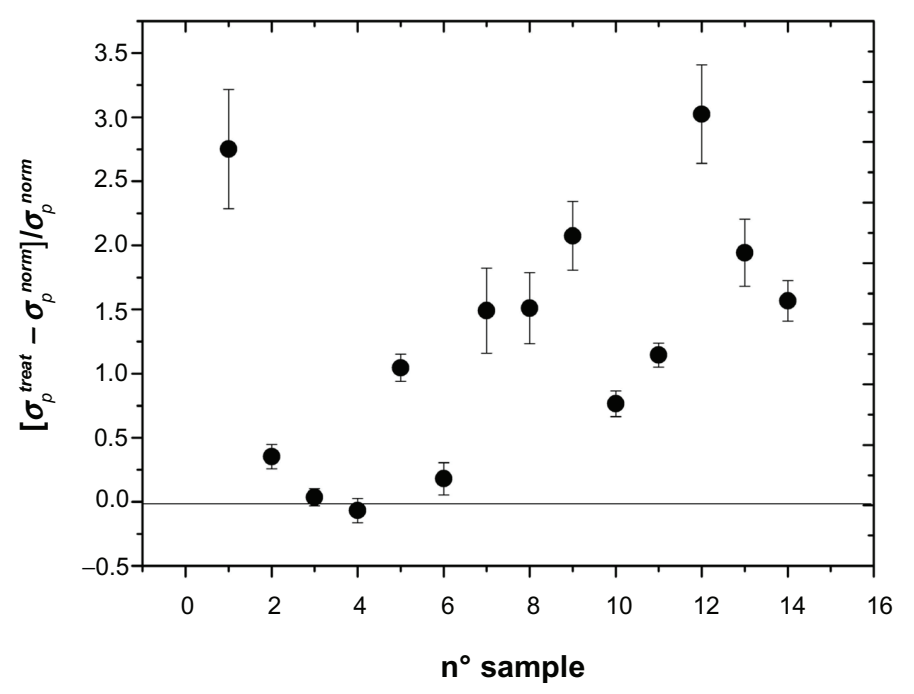

Figure 4 Change in permittivity of the cytosol $\left(\sigma_{p}\right)$ normalized to untreated value: $\left[\sigma_{p}^{\text {teot }}-\sigma_{p}^{\text {nomm }}\right] / \sigma_{p}^{\text {nomm }}$ of the cytosol of erythrocytes in different patients treated for carotid artery stenting.

Note: Absence of any alteration is indicated by the horizontal line.

A

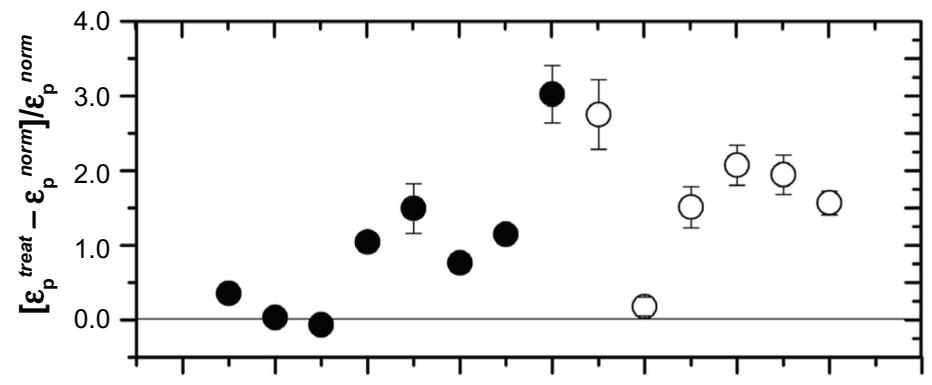

B

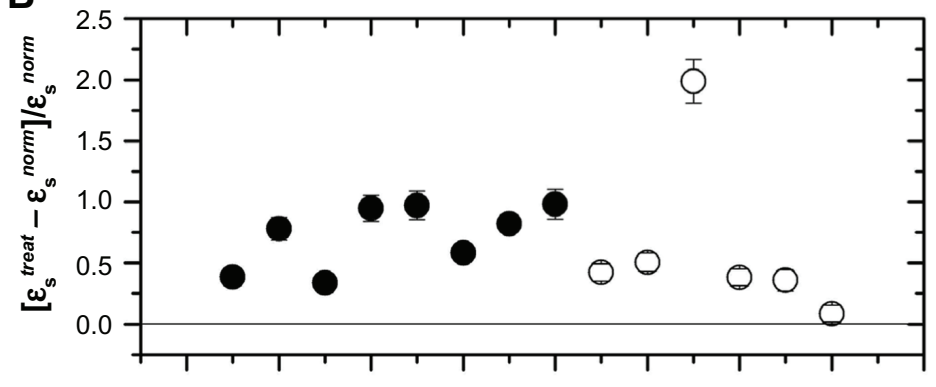

C

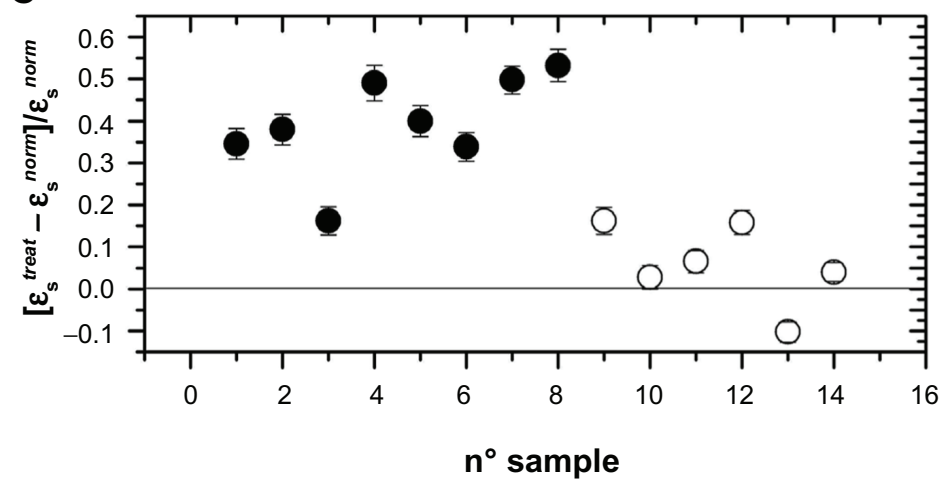

Figure 5 Changes in electrical parameters of the erythrocyte membrane occurring in the first 4 months from stent insertion (full symbols) and in the subsequent 4 months (open symbols). (A) Permittivity changes of intracellular medium (cytosol), (B) membrane conductivity changes, and (C) membrane permittivity changes. 
concluded, on the basis of an analysis of variance followed by a SNK test, that no difference occurred between 8 days and 30 days of treatment. In the present experiment, we extended the temporal interval to 8 months, by dividing the patients into two groups, the first one with $n=8$ considered within the first 4 months after stent insertion and the second one with $n=6$ considered from months 4 to 8 . Changes in the electrical parameters, ie, membrane permittivity $\left(\varepsilon_{s}\right)$, membrane conductivity $\left(\sigma_{s}\right)$, and conductivity of the cytosol $\left(\sigma_{p}\right)$, are shown in Figure 5. Analysis of variance showed a significant difference between the groups $(P<0.01)$, and the SNK test showed significant differences $(P<0.01)$ between controls and the first 4 months of treatment and between controls and the second 4 months of treatment, while no difference was found between the two groups for the first 4 months and the subsequent 4 months of treatment. This means that these alterations persist over a long period of time, and for at least 8 months from stent insertion.

The reliability of the differences observed in the electrical parameters of the erythrocyte membrane based on the fitting algorithm we performed was checked further, considering the influence of electrical conductivity of the extracellular medium on estimates of the fitting parameters. Moreover, we measured, by means of optical microscopy, the size of the erythrocytes and their concentration in suspension using a Coulter cell analyzer. This information contributes to improve the quality of the fitting procedure, which is ultimately reflected in increased reliability of the electrical parameters of the cell membrane.

No pharmacological treatment was considered necessary for the patients after stent insertion and in particular no antiplatelet drugs were administered. Consequently, the observed alterations cannot be attributed to drug consumption. However, many factors could contribute to justify the observed alterations in electrical parameters. In particular, the increase in membrane conductivity $(\sigma)$ is reflected in a decreased surface charge density at the outer cell membrane interface (surface density is about $-3 \mu \mathrm{C} / \mathrm{m}^{2}$ in normal erythrocytes), ${ }^{22}$ playing a significant role in affecting the flow behavior of erythrocytes in the circulation. Because of the re-endothelialization process, we expected, at least in principle, that erythrocyte membrane-prosthesis interactions should decrease as a function of time. On the contrary, the effects we observed persisted for at least 8 months after implantation.

Finally, as pointed out by Barbucci et $\mathrm{al}^{23}$ psychological stress may play a role in patients requiring a cardiovascular prosthesis, in which case any alterations would not be due to a direct effect of stent insertion. In any case, these alterations might compromise the effective biocompatibility of the stent.

\section{Conclusion}

The principal aim of this research was to focus attention on use of a dielectric relaxation spectroscopy technique in characterization of the passive electrical properties of biological cell membranes when they are in close contact with artificial devices. By means of this technique, the membrane permittivity $\left(\varepsilon_{s}\right)$ and membrane conductivity $\left(\sigma_{s}\right)$ of the cell membrane can be properly evaluated, providing information on both the structural and functional behavior of the membrane itself. Any alteration induced by the environment, with which the cell is in close contact, can be measured, and these parameters, in the event that they remain unaltered, can be considered as an index of the biocompatibility of the biomaterial used.

We evaluated possible alterations induced by stents used in the correction of carotid artery stenosis and observed that statistically significant differences occurred in passive electrical parameters of the erythrocyte membrane. These alterations suggest that full biocompatibility has not yet been reached. Despite the positive long-term results of metallic stents, further efforts must be directed towards achievement of ideal biocompatibility of stent materials, ie, an inert material without chemical reactions involving human cells. Our results suggest that some caution is needed. In view of the current paucity of information on the basic mechanisms of interactions of stents with biological cells at a microscopic level, this approach, based on the characterization of the electrical properties of the plasma membrane by means of radiowave dielectric spectroscopy, opens up new possibilities for improving the biocompatibility of biomaterials in biological applications.

\section{Disclosure}

The authors report no conflicts of interest in this work.

\section{References}

1. Hoffman AS. Advances in Chemical Series. Washington, DC: American Chemical Society; 1982.

2. Ratner BD, Hoffman AS, Schoen FD, Lemons JE. Biomaterials Science, An Introduction to Materials in Medicine. Amsterdam, The Netherlands: Elsevier; 2004.

3. Bertrand OF, Sipehia R, Mongrain R, et al. Biocompatibility aspects of new stent technology. J Am Coll Cardiol. 1998;32:562-571.

4. Pethig R. Dielectric and Electronic Properties of Biological Materials. New York, NY: Wiley \& Sons Ltd; 1979

5. Asami K. Characterization of heterogeneous systems by dielectric spectroscopy. Prog Polym Sci. 2002;27:1617-1659. 
6. Hoeber P. A method of measuring electrical conductivity in the interior of cells. Arch Gen Physiol. 1910;133:237-259.

7. Feldman Y, Ermolina I, Hayashi Y. Time domain dielectric spectroscopy study of biological systems. IEEE Trans Diel Elect Ins. 2003;10(5): 728-753.

8. Smith G, Dufy AP, Shen J, Olliff CJ. Dielectric relaxation spectroscopy and some applications in the pharmaceutical sciences. J Pharm Sci. 1995;84:1029-1044.

9. Foster KR, Schwan HP. Dielectric properties of tissues. In: Polk C, Postow E, editors. Handbook of Biological Effects of Electromagnetic Fields. Boca Raton, FL: CRC Press; 1996.

10. Basoli A, Bordi F, Cametti C, Gili T. Structural alteration of erythrocyte cell membrane in presence of artificial prostheses: a radiowave dielectric spectroscopy study. J Biomed Mater Res. 2002;59:100-109.

11. Basoli A, Bordi F, Cametti C, et al. Are aortic endograft prostheses fully hemocompatible? A dielectric spectroscopy investigation of the electrical alterations induced on erythrocyte cell membranes. J Biomed Mater. 2007;2:1-6.

12. Basoli A, Cametti C, Faraglia V, Gili T, Rizzo L, Taurino M. Hemocompatibility of carotid artery stents: alterations of the electrical parameters of erythrocyte cell membrane. a word of caution. Vasc Endovasc Surg. 2010;44:190-197.

13. Hobson RW, Mackey WC, Ascher E. Management of arteriosclerosis carotid artery disease: clinical practice guidelines of the Society for Vascular Surgery. J Vasc Surg. 2008;48:480-486.

14. Rothwell PM. Medical and surgical management of symptomatic carotid stenosis. Int J Stroke. 2006;1:140-149.
15. Grant E, Sheppard R, South G. Dielectric Behaviour of Biological Molecules in Solution. Oxford, UK: Clarendon Press; 1978.

16. Takashima S. Electrical Properties of Biopolymers and Membranes. Bristol, UK: Adam Hilger; 1989.

17. Di Biasio A, Cametti C. On the dielectric relaxation of biological cell suspensions: the effect of membrane electrical conductivity. Colloids Surf B Biointerfaces. 2011;84:433-441.

18. Di Biasio A, Cametti C. D-glucose-induced alterations on the electrical parameters of human erythrocyte cell membrane. Bioelectrochemistry. 2010;77:151-157.

19. Di Biasio A, Ambrosone L, Cametti C. Numerical simulations of dielectric spectra of non-spherical differently-shaped biological cells. J Phys D Appl Phys. 2009;42:025401-025409.

20. Asami K, Hanai T, Koizumi N. Dielectric approach to suspensions of ellipsoidal particles covered with a shell in particular references to biological cells. Japan J Appl Phys. 1980;19(2):359-365.

21. Bordi F, Cametti C, Misasi R, De Persio R, Zimatore G. Conductometric properties of human erythrocyte membrane: dependence of hematocrit and alkali metal ions of suspending medium. Eur Biophys $J$. 1997;26:215-225.

22. Godin C, Caprani A. Effect of blood storage on erythrocyte-wall interactions: implications for surface charge and rigidity. Eur Biophys $J$. 1997;26:175-182.

23. Barbucci R, Lampon Si, Aloisi AM. Effect of behavioral stress on platelet reactivity on polymeric surfaces. J Biomed Mater Res. 1999;48: 9-12.
Vascular Health and Risk Management

\section{Publish your work in this journal}

Vascular Health and Risk Management is an international, peerreviewed journal of therapeutics and risk management, focusing on concise rapid reporting of clinical studies on the processes involved in the maintenance of vascular health; the monitoring, prevention and treatment of vascular disease and its sequelae; and the involvement of

\section{Dovepress}

metabolic disorders, particularly diabetes. This journal is indexed on PubMed Central and MedLine. The manuscript management system is completely online and includes a very quick and fair peer-review system, which is all easy to use. Visit http://www.dovepress.com/ testimonials.php to read real quotes from published authors. 